Richtungen ausgesandt werden, in denen die Gitteratome im Kristall weniger dicht gepackt sind und zu denen dementsprechend eine höhere Fokussierungsenergie gehört. Genauere Auskunft hierüber wird man höchstwahrscheinlich erhalten, wenn man bei der Zerstäubung eines Einkristalls Geschwindig- keitsmessungen in Abhängigkeit von der Richtung der ausgesandten Teilchen durchführt. Entsprechende Experimente werden von uns vorbereitet.

Herrn Prof. Dr. W. Riezler danken wir für sein förderndes Interesse an dieser Arbeit und für viele anregende Diskussionen.

\title{
NOTIZEN
}

\section{Zur Spiegelsymmetrie der Absorptions- und Lumineszenzbanden von Farbstoffen}

Von A. Kawski und B. Polacka

Physikalisches Institut der Pädagogischen Hochschule, Gdańsk (Polen)

(Z. Naturforschg. 17 a, 352 - 354 [1962]; eingegangen am 12. Februar 1962)

Die Spiegelsymmetrie der Absorptions- und Lumineszenzbanden der Farbstoffmoleküle wurde sowohl von Nichols und Merritt ${ }^{1}$ als auch von Lewschin ${ }^{2}$ entdeckt. Stepanow und Kasatschenko ${ }^{3}$ haben gezeigt, daß in den meisten Arbeiten, die der Spiegelsymmetrie der Absorptions- und Emissionsspektren gewidmet sind, die experimentellen Ergebnisse nicht richtig bearbeitet wurden, was oft zu falschen Schlußfolgerungen führte. Schon Blochinzew ${ }^{4}$ und später Förster ${ }^{5}$ haben festgestellt, daß es zweckmäßig ist, für die Absorptionsspektren auf der Ordinate die Werte $\psi_{v} / v$ und für die Lumineszenzspektren die Werte $W_{\nu}^{\mathrm{Lum}} / \nu^{4}\left(\varkappa_{v}\right.$ ist hier der Absorptionskoeffizient, $W_{v}$ Lum die Lumineszenzleistung) aufzutragen. In diesem Fall entspricht die Spiegelsymmetrie der Symmetrie der Matrixelemente für die Dipolmomente der Elektronen-Oszillationsübergänge.

In der vorliegenden Arbeit wurden Absorptions- und Emissionsspektren verschiedener organischer Farbstoffe in flüssigen und festen Lösungsmitteln nach der früher ${ }^{6}$ beschriebenen Methode gemessen. Die Meßergebnisse für Rhodamin $6 \mathrm{G}$, Rhodamin B und gelbliches Eosin im Mono- und Polymethylmethakrylat und in Glycerin

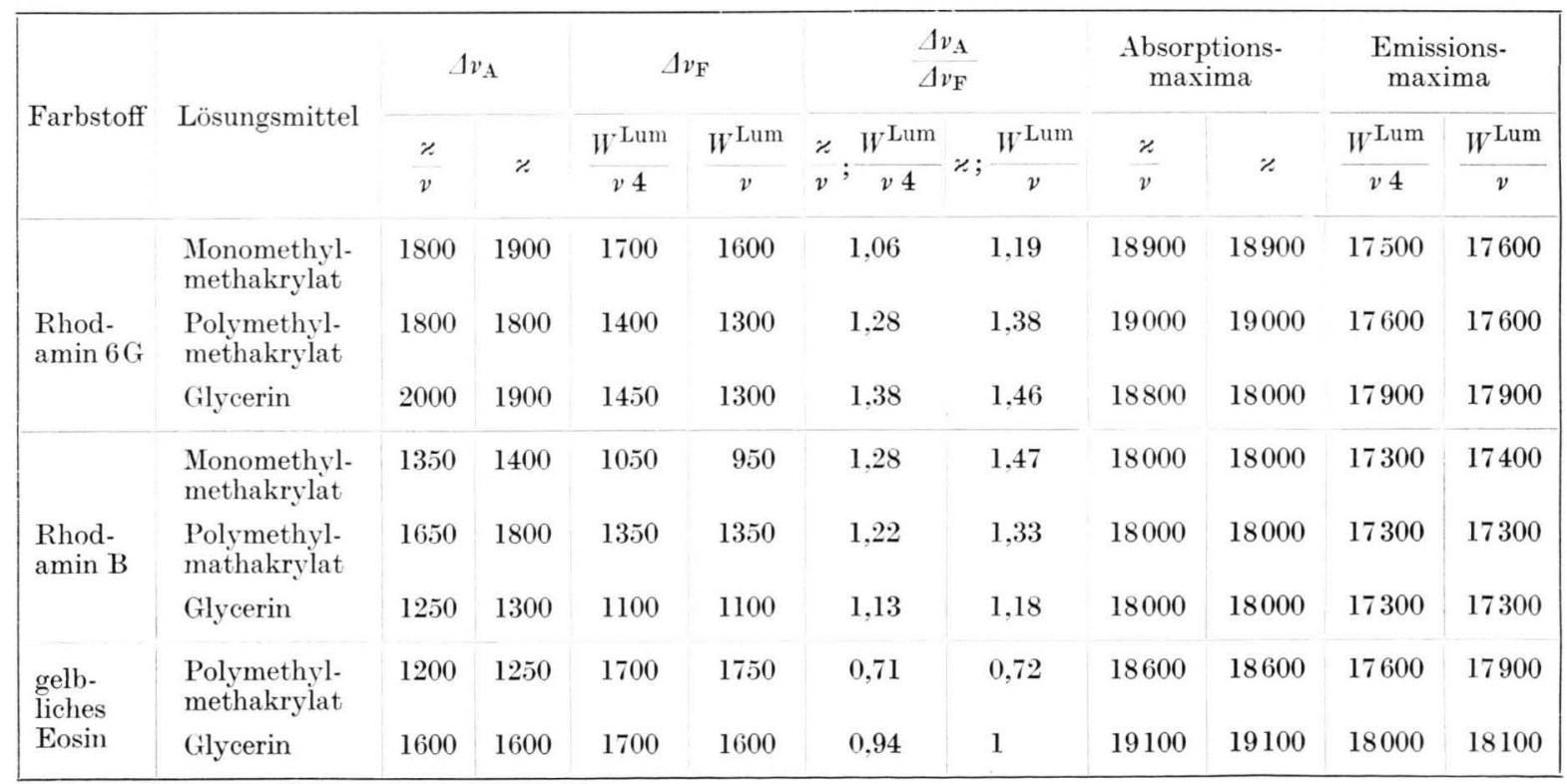

Tab. 1. Halbwertsbreiten $\Delta v$ (in $\mathrm{cm}^{-1}$ ) der Absorptions- und Fluoreszenzbanden für Farbstoffe in flüssigen und festen Lösungen bei Raumtemperatur.

1 E. L. Nichols u. E. Merritt, Phys. Rev. 30, 328 [1910].

2 W. L. Lewschin, Z. Phys. 72, 368 [1931].

3 L. P. Kasatschenko u. B. J. Stepanow, Opt. i Spektr. 2, 339 [1957].

4 D. Blochinzew, J. Physics USSR 1, 117 [1939].
5 TH. Förster, Fluoreszenz organischer Verbindungen, Vandenhoek u. Rupprecht, Göttingen 1951.

6 A. Kawski, B. Polacka u. Z. Polacki, Acta Phys. Polon. 20, 903 [1961]. 
(die Lösungsmittel enthielten zur Verbesserung ihrer Lösungsfähigkeit 10\% Äthylalkoholzusatz) sind in Abb. 1-8 dargestellt, wobei auf der Ordinate $\kappa_{v} / v$ für die Absorptionsspektren und $W_{v} \mathrm{Lum} / v^{4}$ für die Lumineszenzspektren aufgetragen wurden. In Tab. 1 sind die Halbwertsbreiten $\Delta v$ in $\mathrm{cm}^{-1}$ und die Absorptions- und Fluoreszenzbandenmaxima der genannten Farbstofflösungen, mit der neuen und mit der alten Methode bearbeitet, zusammengestellt. Aus der Tabelle ist ersichtlich, daß in allen untersuchten Fällen keine sichtbare Maximaänderung der Absorptions- und Emissionsbanden stattfindet. Eine Halbwertsbreitenänderung der Absorptions- und Emissionsbanden ist allerdings wahrnehmbar. Da für Farbstoffe (z. B. Rhodamin $6 \mathrm{G}$ in Äthanol) diese Änderung nicht groß war, ist es LEwschin gelungen, eine Symmetrie der Absorptions- und

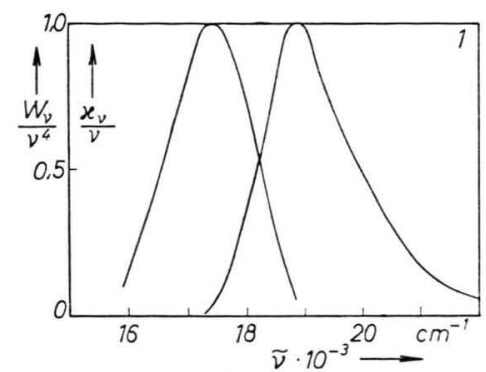

Abb. 1. Absorptions- und Emissionsspektren des Rhodamins $6 \mathrm{G}$ in Monomethylmethakrylat.
Emissionsbanden festzustellen. Aus der Tabelle geht weiter hervor, daß eine exakte Spiegelsymmetrie der Absorptions- und Lumineszenzspektren nur in wenigen Fällen beobachtet wird. Es ist zu sehen, daß eine Abweichung von der Symmetrie, in Übereinstimmung mit der Stepanowschen Feststellung ${ }^{3}$, keine Ausnahme bildet, was mit einer Nichterfüllung der folgenden Bedingung verbunden ist:

$$
\begin{gathered}
\int_{E_{\mathrm{s}}} c g\left(E_{\mathrm{s}}\right) \exp \left(-\frac{E_{\mathrm{s}}}{k T}\right) B_{\mathrm{Abs}}\left(E_{\mathrm{s}}, v_{\mathrm{Abs}}\right) \mathrm{d} E_{\mathrm{s}} \\
=\int_{E_{\mathrm{s}}{ }^{*}} c^{*} g^{*}\left(E_{\mathrm{s}}{ }^{*}\right) \exp \left(\frac{E_{\mathrm{s}}{ }^{*}}{k T}\right) B_{\mathrm{Em}}\left(E_{\mathrm{s}}{ }^{*}, v_{\mathrm{Lum}}\right) \mathrm{d} E_{\mathrm{s}}{ }^{*},
\end{gathered}
$$

wobei $c$ und $c^{*}$ Normalisierungskonstanten, $g\left(E_{\mathrm{s}}\right)$ und

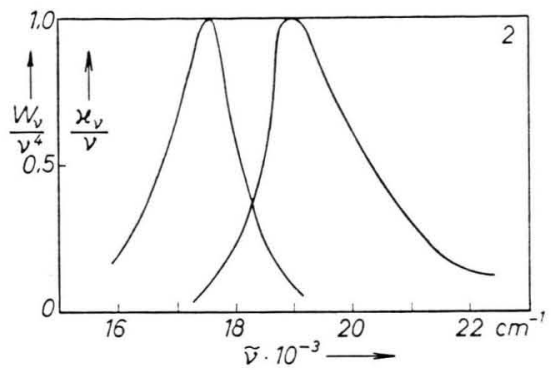

Abb. 2. Absorptions- und Emissionsspektren des Rhodamins $6 \mathrm{G}$ in Polymethylmethakrylat.

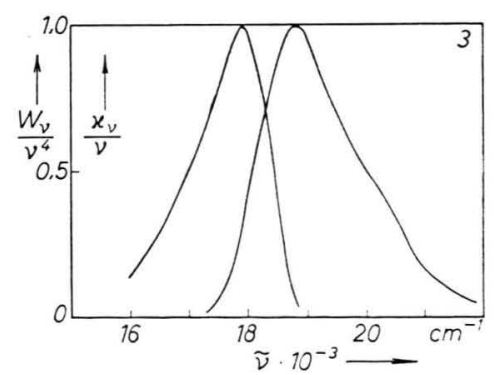

Abb. 3. Absorptions- und Emissionsspektren des Rhodamins $6 \mathrm{G}$ in Glycerin.

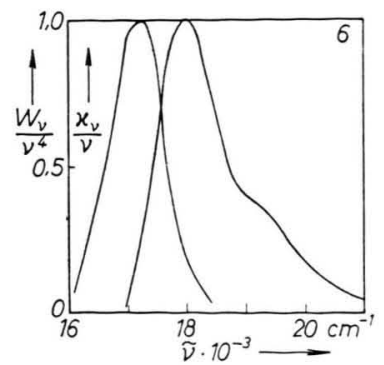

Abb. 6. Absorptions- und Emissionsspektren des Rhodamins B in Glycerin.

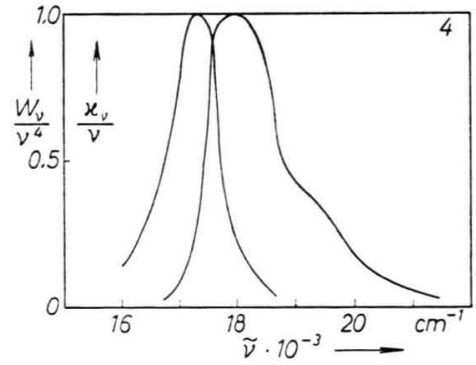

Abb. 4. Absorptions- und Emissionsspektren des Rhodamins B in Monomethylmethakrylat.

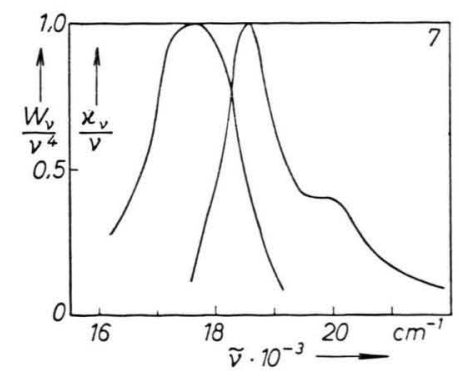

Abb. 7. Absorptions- und Emissionsspektren des gelblichen Eosins in Poly. methylmethakrylat.

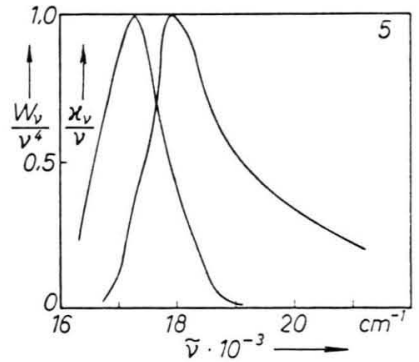

Abb. 5. Absorptions- und Emissionsspektren des Rhodamins B in Poly. methylmethakrylat.

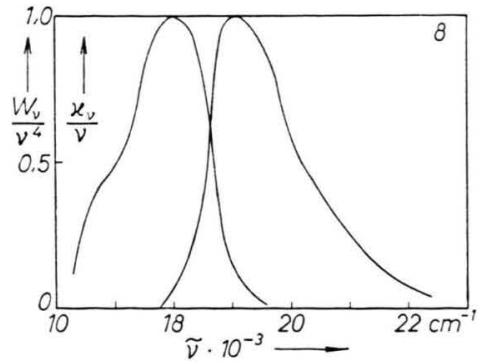

Abb. 8. Absorptions- und Emissionsspektren des gelblichen Eosins in Gly. cerin. 
$g^{*}\left(E_{\mathrm{s}}{ }^{*}\right)$ statistische Gewichte der Schwingungs-Energieniveaus $E_{\mathrm{s}}$ und $E_{\mathrm{s}}{ }^{*}, k$ die Boltzmann-Konstante und $T$ die absolute Temperatur bedeuten.

Der Ausdruck (1) stellt die vollkommene Bedingung der Spiegelsymmetrie für die Absorptions- und Emissionsspektren der zusammengesetzten Moleküle dar. Die Bedingung (1) ist erfüllt, wenn

$$
g^{*}\left(E_{\mathrm{S}}{ }^{*}\right)=g\left(E_{\mathrm{s}}\right) \text { bei } E_{\mathrm{S}}{ }^{*}=E_{\mathrm{S}} \text { und } c^{*}=c,
$$

und wenn für symmetrische Übergänge die Gleichheit der Absorptionswahrscheinlichkeit $B_{\mathrm{Abs}}$ mit der Wahrscheinlichkeit der erzwungenen Emission $B_{\mathrm{Em}}$ erfüllt ist.
Für gelbliches Eosin im Polymethylmethakrylat wird, ähnlich wie im Äthylalkohol, eine Überlagerung von zwei Banden beobachtet, was wahrscheinlich auf zwei verschiedene Ionenformen zurückzuführen ist.

Da das absorbierende oder emittierende Molekül einer Wechselwirkung mit den umgebenden Lösungsmittelmolekülen unterworfen ist, sind die beobachteten Absorptions- und Lumineszenzspektren (Abb. 1-8) für die Eigenschaften des Systems Farbstoffmolekül-Lösungsmittel und nicht für die des emittierenden Moleküls kennzeichnend.

\section{Spindichten im Cyclohexadienylradikal}

\section{Von H. Fischer}

Deutsches Kunststoff-Institut, Darmstadt

(Z. Naturforschg. 17 a, 354 [1962]; eingegangen am 3. März 1962)

In einer vorausgehenden Arbeit ${ }^{1}$ wurde das Elektronenspinresonanzspektrum des bei $77^{\circ} \mathrm{K}$ bestrahlten Benzols durch das Cyclohexadienylradikal interpretiert. Unter den Annahmen, daß dieses Radikal, wie offenbar alle "odd alternant hydrocarbon radicals" ${ }^{2-4}$, alternierende Vorzeichen der Spindichten längs der Kette von trigonalen Kohlenstoffatomen aufweist und daß die Spindichtefunktion in erster Näherung im Bereich der Methylengruppe verschwindet, kann man aus den dem Spektrum entnommenen Kopplungskonstanten folgende „experimentelle“ Spindichten in den $2 \mathrm{p}_{z}$-Eigenfunktionen der ortho-, para- und meta-ständigen Kohlenstoffe ermitteln :

$$
\varrho_{0} \approx \varrho_{\mathrm{p}} \approx 0,40, \quad \varrho_{\mathrm{m}} \approx-0,10 .
$$

Im folgenden werden die Ergebnisse von Berechnungen "theoretischer" Werte dieser Spindichten dargelegt. Wir haben nach verschiedenen bekannten Verfahren die Eigenfunktion des $\pi$-Elektronensystems aufgebaut und daraus die Spindichteverteilung ${ }^{3}$ bestimmt. Grundsätzlich wurden dabei die $\pi$ - $\sigma$-Wechselwirkung, der Einfluß der Methylengruppe und die Nichtorthogonalität der $2 \mathrm{p}_{z}$-Kohlenstoffeigenfunktionen nicht berücksichtigt.

a) Unter Vernachlässigung ionischer Strukturen erhält man nach der Methode der Valenzstrukturen (v.b.) die Werte

$$
\varrho_{0}=0,512, \quad \varrho_{\mathrm{p}}=0,566, \varrho_{\mathrm{m}}=-0,295,
$$

wenn man Strukturen mit langen Bindungen verwendet, aber

$$
\varrho_{0}=0,608, \quad \varrho_{\mathrm{p}}=0,667, \quad \varrho_{\mathrm{m}}=-0,441,
$$

wenn man diese zum Aufbau der Eigenfunktion nicht mitbenutzt.

b) Die verkürzte Methode der Molekülzustände (MOLCAO) liefert

$$
\varrho_{0}=\varrho_{\mathrm{p}}=; \quad \varrho_{\mathrm{m}}=0 .
$$

c) Unter Anwendung der von Lefebvre u. Mitarb. ${ }^{4}$ eingeführten Verfeinerung der Methode der Molekülzustände durch semi-empirische Berücksichtigung der Konfigurationswechselwirkung gelangen wir zu den folgenden Gleichungen für die Spindichten in Abhängigkeit von dem Wechselwirkungsparameter

$$
0<q=\operatorname{tg} \Theta<1
$$

$$
\begin{aligned}
& \varrho_{0}=\frac{1}{N}\left(\frac{1}{3}+\frac{4}{9} q+\frac{4}{27} q^{2}+\frac{20}{27} q^{3}+\frac{10}{9} q^{4}+\frac{20}{27} q^{5}+\frac{4}{27} q^{6}+\frac{4}{9} q^{7}+\frac{1}{3} q^{8}\right) \\
& \varrho_{\mathrm{p}}=\varrho_{\mathrm{o}}, \\
& \varrho_{\mathrm{m}}=\frac{1}{N}\left(-\frac{2}{3} q+\frac{4}{9} q^{2}-\frac{10}{9} q^{3}-\frac{10}{9} q^{5}+\frac{4}{9} q^{6}-\frac{2}{3} q^{7}\right) \quad \text { mit } \quad N=1+\frac{4}{3} q^{2}+\frac{10}{3} q^{4}+\frac{4}{3} q^{6}+q^{8} .
\end{aligned}
$$

Setzt man den experimentellen Wert $\varrho_{0}=0,40$ in Gl. (5) ein, so ist $\operatorname{tg} \Theta=q=0,167$ eine vernünftige Wurzel der ersten dieser Gleichungen, und es werden

$$
\varrho_{\mathrm{o}} \equiv \varrho_{\mathrm{p}}=0,40, \quad \varrho_{\mathrm{m}}=-0,10 .
$$

Insbesondere die nach den genaueren Näherungsverfahren gewonnenen Werte (2) und (6) sind im Hinblick

${ }^{1}$ H. Fischer, Kolloid-Z. 180, 64 [1962].

2 H. M. McConnell u. D. B. Chesnut, J. Chem. Phys. 27, 984 [1957]. auf die der Rechnung zugrunde liegenden Vernachlässigungen in guter Übereinstimmung mit den „experimentellen" Spindichten (1). Damit liefern sie die erhoffte theoretische Stütze für die Interpretation des Spektrums.

Ich danke Herrn Professor Dr. K. H. Hellwege für anspornende Unterstützung dieser Arbeit.

3 H. M. McConnell, J. Chem. Phys. 28, 1188 [1958].

4 R. Lefebvre, H. H. Dearman u. H. M. McConnele, J. Chem. Phys. 32, 176 [1960]. 